\title{
Gene Regulation of Catecholamine Biosynthetic Enzymes by Nitric Oxide in PC12 Cells
}

\author{
Dominique Ansell1,2, Julie Grandbois ${ }^{1,2}$, T. C. Tai ${ }^{1,2,3,4}$ \\ ${ }^{1}$ Medical Sciences Division, Northern Ontario School of Medicine, Sudbury, Canada \\ ${ }^{2}$ Department of Biology, Laurentian University, Sudbury, Canada \\ ${ }^{3}$ Department of Chemistry \& Biochemistry, Laurentian University, Sudbury, Canada \\ ${ }^{4}$ Biomolecular Sciences, Laurentian University, Sudbury, Canada \\ Email: tc.tai@nosm.ca
}

Received 7 March 2014; revised 2 April 2014; accepted 9 April 2014

Copyright (C) 2014 by authors and Scientific Research Publishing Inc.

This work is licensed under the Creative Commons Attribution International License (CC BY).

http://creativecommons.org/licenses/by/4.0/

(c) (i) Open Access

\section{Abstract}

Nitric oxide (NO) regulates a wide range of physiological processes. Recent studies show that NO can regulate the release of catecholamines (CA) from the adrenal medulla. In the current study, the PC12 cell line was used to examine the effect of NO on the regulation of the CA biosynthetic enzymes: tyrosine hydroxylase (TH), dopamine- $\beta$-hydroxylase (DBH) and phenylethanolamine $\mathrm{N}$ methyltransferase (PNMT). Treatment of PC12 cells with the NO donor, sodium nitroprusside (SNP) for 6 hours significantly increased TH, DBH and PNMT mRNA levels. In addition, NO potentiates the regulation of gene expression of all three CA biosynthetic enzymes by glucocorticoids and cholinergic agonists. The signaling pathways involved in NO regulation of CA biosynthetic enzymes were investigated with the use of specific kinase activators and inhibitors, with results supporting a contributing role of PKA, PKC and PKG in SNP-mediated induction for all three CA genes ( $p<$ 0.01). In addition, inhibitors of transcription and translation inhibited SNP-mediated induction of all three genes $(p<0.001)$ suggesting that both transcriptional and translational mechanisms may be involved in CA gene regulation by NO. Results from this study show that in addition to regulating CA biosynthetic enzymes, NO can also potentiate cholinergic and glucocorticoid activation of CA genes.

\section{Keywords}

NO, PC12 Cells, TH, DBH, PNMT, Catecholamines 


\section{Introduction}

Nitric oxide (NO) is a volatile gas that has been associated with a wide range of physiological processes. First identified as an endothelial derived factor (EDRF) involved in blood vessel relaxation, NO has recently been recognized as an important intercellular messenger in the central and peripheral nervous systems, involved in events such as neurotransmitter release, long-term potentiation and gene transcription [1] [2]. NO is synthesized from L-arginine and molecular oxygen by nitric oxide synthase (NOS) which exists in various isoforms including neuronal (nNOS), endothelial (eNOS) and inducible (iNOS) [2]. Each NOS isoform has a specific distribution profile in the body including the adrenal gland [3]. The numerous physiological effects of NO are mediated by elevation of intracellular cGMP levels through activation of soluble guanylate cyclase (sGC) [3] [4].

The complete L-arginine/NO/cGMP pathway exists in the adrenal medulla, a tissue that is critically important in the regulation, synthesis and release of catecholamines (CA) during stress response [3] [5]. NOS is present in the adult rat adrenal gland as well as in CA producing bovine chromaffin cells [3]. NO can regulate adrenal cortical and medullary functions such as secretion of aldosterone, corticosterone and catecholamines (CAs) in the adrenal gland [6]. Recent studies show that NO increases the synthesis of adrenal CAs via induction of tyrosine hydroxylase $(\mathrm{TH})$, dopamine $\beta$-hydroxylase $(\mathrm{DBH})$ and phenylethanolamine $\mathrm{N}$-methyltransferase (PNMT) via the cGMP-dependent signaling pathway [1]. SNP-derived NO has also been shown to increase TH activity and the levels of norepinephrine and epinephrine in the adrenal medulla of rats [7].

In-vivo, CA synthesis is mediated by hormonal regulation by the hypothalamic-pituitary-adrenal (HPA) axis via secretion of glucocorticoids and by neural regulation via the sympatho-adrenal (SA) system where the splanchnic nerve synthesizes and secretes a number of neurotransmitters, including acetylcholine [8] [9]. Activation of the HPA induces guanosine triphosphate cyclohydrolase I levels associated with NO biosynthesis [10]. NO can also activate the SA system, via cholinergic stimulation, to enhance the CA biosynthetic pathway [7]. Furthermore, NO-induced elevations in cGMP may have a synergistic action on the TH, DBH and PNMT activating mechanisms of nicotinic stimulation [7].

The current study provides evidence that NO is capable of regulating genes encoding for the CA biosynthetic enzymes in the PC12 cell culture model. Furthermore, results demonstrate that NO is also capable of potentiating both neural and hormonal regulation of CA biosynthetic enzymes.

\section{Methods}

\subsection{Cell Culture}

PC12 cells (Dr. O'Connor, UCSD, San Diego, CA, USA) were cultured in Dulbeco's modified Eagle's medium (DMEM) (Hyclone, Thermo Fisher Scientific, Nepean, ON, Canada) supplemented with 5\% equine serum, 5\% bovine calf serum (Hyclone Inc., Logan, UT, USA) and gentamycin sulphate $(50 \mu \mathrm{g} / \mathrm{mL})$ (Fisher Scientific, Ottawa, ON, Canada). Cells were maintained in a humidified incubator at $37^{\circ} \mathrm{C}$ in an atmosphere of $5 \% \mathrm{CO}_{2}-95 \%$ air and grown to $80 \%$ - 90\% confluency before being passed or used in an experiment. Prior to experimentation, cells were washed twice with PBS, trypsinized, and transferred to DMEM containing charcoal-treated serum. For RNA extraction, cells were seeded in $60 \mathrm{~mm}$ culture plates at a density of $1.6 \times 10^{6}$ cells $/ \mathrm{mL}$. Following seeding, cells were allowed to adhere to plates for $16-24 \mathrm{~h}$ prior to beginning experiments.

PC12 cells were treated with the NO donor sodium nitroprusside (SNP) to assess the effects of NO on the regulation of the CA biosynthetic enzymes. To determine the impact of SNP on cholinergic regulation of CA enzymes, cells were treated with a combination of SNP with either $100 \mu \mathrm{M}$ nicotine (Nic), muscarine (Mus) or carbamylcholine $(\mathrm{CCh})$. To determine whether SNP potentiates the hormonal activation of CA enzymes, cells were treated with SNP in combination with $1 \mu \mathrm{M}$ dexamethasone (DEX). The effects of NO on intracellular kinase activators of CA enzymes was investigated by treating cells with $10 \mu \mathrm{M}$ forskolin (Fn) (PKA activator), $80 \mathrm{nM}$ PMA (PKC activator) and $100 \mu \mathrm{M}$ 8-Br-cGMP (cGMP-PKG activator) in combination with SNP. In subsequent experiments, cells pre-treated with the inhibitors for PKA (30 $\mu \mathrm{M} \mathrm{H}-89)$, PKC (100 nM GF109203X), cGMP (200 nM A6563) and PKG (100 nM DT-2) for $30 \mathrm{~min}$ prior to treatments with SNP. To assess whether the mRNA induction caused by SNP could be attenuated by inhibitors of transcription and translation, cells were pre-treated with $50 \mathrm{nM}$ actinomycin-D (actino-D) and $1 \mu \mathrm{M}$ cycloheximide (cyclohex) respectively and a combination of each inhibitor with SNP. All reagents were purchased from Sigma Aldrich (Oakville, ON, Canada). 


\subsection{RT-PCR}

Total RNA was isolated from PC12 cells using TRI Reagent (Sigma Aldrich Canada) according to the manufacturer's instructions. Total RNA pellets were resuspended in diethylpyrocarbonate-treated water and stored at $-80^{\circ} \mathrm{C}$. Prior to cDNA synthesis, $2 \mu \mathrm{g}$ of total RNA was subjected to deoxyribonuclease I treatment (Sigma Aldrich Canada) to eliminate possible genomic DNA contamination. cDNA synthesis was performed using $100 \mathrm{U}$ Revert Aid Moloney Murine Leukemia Virus reverse transcriptase (Fermentas, Burlington, ON), $0.5 \mu \mathrm{g} / \mu \mathrm{L}$ of random hexamers, $200 \mu \mathrm{mol} / \mu \mathrm{L}$ dNTPs in a total reaction volume of $35 \mu \mathrm{L}$. In selected tubes the reverse transcriptase was omitted as a control of amplification from contaminating cDNA or genomic DNA. The reaction was carried out at $42^{\circ} \mathrm{C}$ for $60 \mathrm{~min}$ and was terminated at $70^{\circ} \mathrm{C}$ for $10 \mathrm{~min}$ in a MG Mini Personal Thermocycler from Biorad (Hercules, CA, USA).

PCR was performed in $25 \mu \mathrm{L}$ reaction volumes containing $78 \mathrm{ng}$ of cDNA, using $50 \mathrm{U}$ GoTaq Flexi DNA polymerase (Promega) containing $200 \mu \mathrm{M}$ of dNTPs, $1.5 \mathrm{mM} \mathrm{MgCl}_{2}$ and $25 \mathrm{ng}$ of forward and reverse primer sequences specific for the following genes: tyrosine hydroxylase (TH) (5'-GCGACAGAGTCTCATCGAGGAT-3' and 5'-AAGAGCAGGTTGAGAACAGCATT-3' for 20 cycles at $52^{\circ} \mathrm{C}$ ), dopamine- $\beta$-hydroxylase (DBH) (5'-GACAGGACCTACTTTGCGAC-3' and 5'-AGCTGTGTAGTGTAGACGGATGC-3' for 27 cycles at $58^{\circ} \mathrm{C}$ ), phenylethanolamine-N-methyltransferase (PNMT) (5'-CAGACTTCTTGGAGGTCAACCG-3' and 5'AGCAGCGTCGTGATATGATAC-3' for 35 cycles at $58^{\circ} \mathrm{C}$ ) and glyceraldehyde 3-phosphate dehydrogenase (GAPDH) (5'-ATGGTGGTGCTGAGTATGTCG-3' and 5'-CATGTCAGATCCACAACGGATAC-3' for 21 cycles at $\left.58^{\circ} \mathrm{C}\right)$. PCR products $(10 \mu \mathrm{L})$ were electrophoresed on a $1.5 \%$ agarose gel in $40 \mathrm{mM}$ Tris-acetate and 2 mM EDTA buffer, $\mathrm{pH}$ 8.0; stained with ethidium bromide (EtBr); and documented using Chemidoc XRS (Biorad) imaging system and densitometric analysis performed with Quantity One software (Biorad).

\subsection{Western Blot Analysis}

Total protein was isolated and extracted from PC12 cells by the addition of RIPA lysis buffer $(25 \mathrm{mM}$ Tris $\mathrm{HCl}$, $150 \mathrm{mM} \mathrm{NaCl}, 1 \% \mathrm{NP} 40,1 \%$ sodium deoxycholate, $0.1 \%$ SDS, 2 mM EDTA, $0.5 \mathrm{mM}$ PMSF, protease inhibitors, $\mathrm{pH}$ 7.6). Cell lysates were incubated on ice for $10 \mathrm{~min}$ prior to centrifugation at $12000 \times \mathrm{g}$ for $20 \mathrm{~min}$ at $4^{\circ} \mathrm{C}$, supernatants collected and stored at $-80^{\circ} \mathrm{C}$ until use. Protein concentrations of the extracts were determined by the Bradford method as previously described [8].

Protein samples $(50 \mu \mathrm{g})$ were prepared using RIPA lysis buffer with the addition of $6 \mathrm{x}$ loading dye $(300 \mathrm{mM}$ Tris $\mathrm{HCl}, 12 \%$ SDS, $12 \mathrm{mM}$ EDTA, $6 \% \beta$-mercaptoethanol, $60 \%$ glycerol, $6 \%$ bromophenol blue, $\mathrm{pH} 6.8$ ) and denatured for $2 \mathrm{~min}$ at $95^{\circ} \mathrm{C}$. Samples were resolved on $7 \%$ and $12 \%$ sodium dodecyl sulphate polyacrylamide gel (SDS-PAGE) and resolved for $1.5 \mathrm{~h}$ at $100 \mathrm{~V}$. Proteins were transferred to polyvinylidene fluoride membranes (Whatman) (1.5 h at $100 \mathrm{~V}$ ). Membranes were stained using Ponceau S (Sigma) to confirm equal loading of each sample. Membranes were blocked with 10\% skim milk dissolved in 1X TBS-T (10 mM Tris HCl, 150 $\mathrm{mM} \mathrm{NaCl}, 0.05 \%$ Tween $20, \mathrm{pH} 8.0)$ for one hour, washed $(3 \times 10 \mathrm{~min})$ with TBS-T, then incubated for one hour with the TH, DBH (Novus) or PNMT (Immunostar) primary antibodies. (1:8000 dilution in 10\% skim milk). The GAPDH primary antibody was diluted 1:5000 and incubated overnight. Membranes were washed (3 $\times 10 \mathrm{~min}$ ) with TBS-T, followed by incubation with secondary antibody conjugated with horseradish peroxidase (1:5000 for $45 \mathrm{~min})$. Membranes were then washed $(3 \times 10 \mathrm{~min})$ with TBS-T and enhanced chemiluminescence detection was subsequently performed, followed by autoradiography.

\subsection{Statistical Analysis}

All data are presented as the mean \pm SEM. Experiments were repeated at least three times. Statistical significance between experimental and control groups was determined by one-way ANOVA followed by post hoc comparisons using the Student-Newman-Keuls multiple comparison test. Results were considered statistically significant with values of $\mathrm{p}<0.05$.

\section{Results}

\subsection{Effect of NO on Expression of Genes Associated with Catecholamine Biosynthesis}

RT-PCR analysis from PC12 cells treated for $6 \mathrm{~h}$ with the NO donor (100 $\mu \mathrm{M}$ SNP) significantly increased TH 
mRNA 2.0-fold ( $p<0.01)$ and DBH mRNA levels 1.3-fold $(\mathrm{p}<0.05)$ compared to untreated control (Figure $1(\mathrm{a})$ ). Furthermore, SNP treatment significantly increased both the PNMT intron-retaining $(4.5$-fold; $p<0.001)$ and intronless mRNA splice variants $(2.0$ fold; $\mathrm{p}<0.01)$. Similar increases in protein levels of TH, DBH and PNMT were also detected by western blot analysis (Figure 1(b)).

\subsection{Effect of NO on Cholinergic Regulation of Biosynthetic Enzymes}

To determine the impact of NO elevations on cholinergic regulation of CA biosynthetic enzymes, RT-PCR analysis from PC12 cells treated for $6 \mathrm{~h}$ with specific cholinergic receptor agonists (carbamylcholine, nicotine and muscarine) and SNP was conducted (Figure 2(a)). CCh treatment significantly increased expression of all three genes compared to untreated control $(\mathrm{p}<0.01)$. The addition of SNP in combination with CCh significantly increased TH and PNMT intronless mRNA levels compared to treatments with SNP alone (1.6, 1.6-fold; $\mathrm{p}<0.01$ ), whereas DBH and PNMT intron-retaining mRNA levels were unchanged. Nic treatment significantly increased TH, PNMT intron-retaining and intronless $(p<0.01)$ compared to untreated control, whereas DBH mRNA was unchanged. The addition of SNP in combination with Nic further increased DBH mRNA levels compared to treatments with SNP alone $(1.5$-fold; $p<0.05)$. Mus treatment significantly increased TH and PNMT intronless mRNA levels compared to untreated control $(\mathrm{p}<0.01)$. The addition of SNP in combination with Mus further increased TH and PNMT intronless mRNA levels compared to treatments with SNP alone (1.3, 1.4-fold; $\mathrm{p}<0.01$ ), whereas DBH and PNMT intron-retaining were unchanged.

\subsection{Effect of NO on Hormonal Regulation of Catecholamine Biosynthetic Enzymes}

To determine whether NO can potentiate the glucocorticoid activation of CA biosynthetic enzymes, RT-PCR analysis was performed from PC12 cells treated with $1 \mu \mathrm{M}$ DEX and $100 \mu \mathrm{M}$ SNP for $6 \mathrm{~h}$ (Figure 2(b)). TH, DBH and PNMT intronless mRNA levels were all significantly increased under DEX treatment alone $(\mathrm{p}<$ 0.001). Treatment of cells with SNP in combination with DEX treatment significantly increased mRNA expression of TH, DBH, and PNMT intronless forms compared to treatments with SNP alone (1.2, 1.9, 4.2-fold; $\mathrm{p}<$ $0.001)$.

\subsection{Intracellular Signaling Pathways Involved in NO Regulation of CA Biosynthetic Enzymes}

The intracellular signaling pathways involved in the NO regulation of CA biosynthetic enzymes were investigated with specific kinase activators (Figure 3(a)) and inhibitors (Figure 3(b)). For the specific kinase activators, PC12 cells were treated with $10 \mu \mathrm{M}$ forskolin (PKA activator), $80 \mathrm{nM}$ PMA (PKC activator) and $100 \mu \mathrm{M}$

(a)

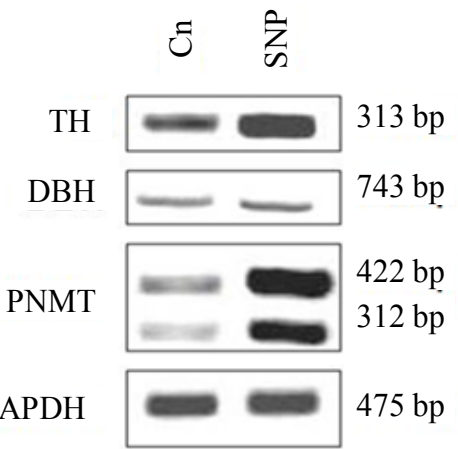

(b)

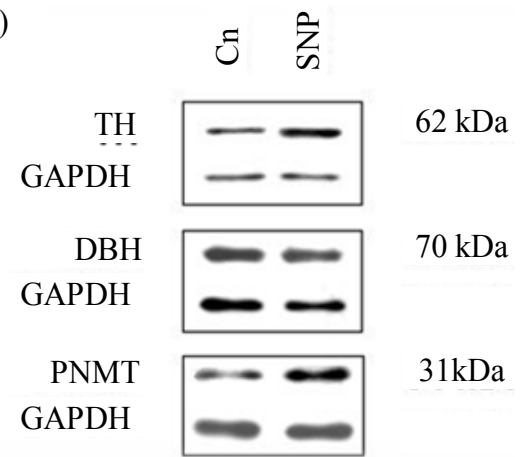

Figure 1. mRNA levels of genes associated with catecholamine biosynthesis following SNP treatment. (a) PC12 cells were treated with $100 \mu \mathrm{M}$ SNP (NO donor) for $6 \mathrm{~h}$. Representative image $(\mathrm{n}=3)$ of TH, DBH and PNMT (intron-retaining and intronless splice variants) mRNA levels from semi-quantitative RT-PCR. Products were resolved on $1.5 \%$ EtBr-agarose gels. GAPDH mRNA was used as a housekeeping control. (b) PC12 cells were treated with $100 \mu \mathrm{M}$ SNP (NO donor) for $6 \mathrm{~h}$. Representative image $(\mathrm{n}=3)$ of TH, DBH and PNMT protein levels from Western Blot analysis. Products were resolved on SDS-PAGE gels. GAPDH primary antibody was used as a housekeeping control. 
(a)

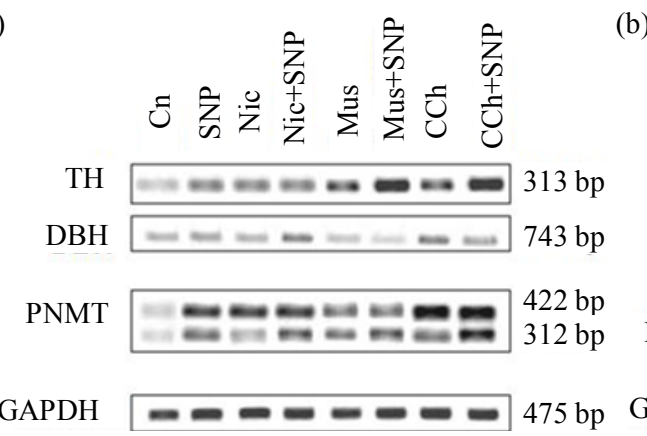

(b)

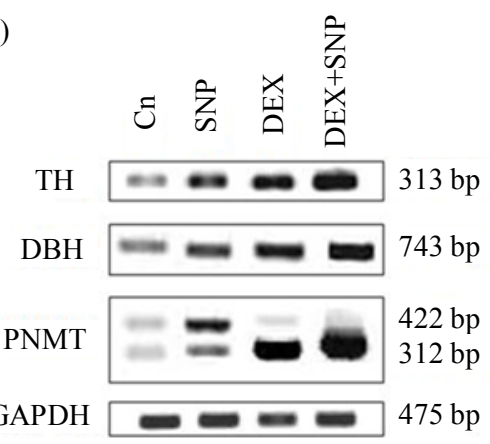

)

Figure 2. mRNA levels of genes associated with catecholamine biosynthesis following cholinergic agonist, glucocorticoid and SNP treatments. (a) PC12 cells were treated with $100 \mu \mathrm{M}$ nicotine, $100 \mu \mathrm{M}$ muscarine, $100 \mu \mathrm{M}$ carbamylcholine, $100 \mu \mathrm{M}$ SNP and the combination of each agonist with SNP for $6 \mathrm{~h}$. (b) PC12 cells were treated with $1 \mu \mathrm{M}$ DEX, $100 \mu \mathrm{M}$ SNP and the combination for $6 \mathrm{~h}$. Representative image $(\mathrm{n}=3)$ of TH, DBH and PNMT (intronretaining and intronless splice variants) mRNA levels from semi-quantitative RT-PCR. Products were resolved on 1.5\% EtBr-agarose gels. GAPDH mRNA was used as a housekeeping control.

(a)

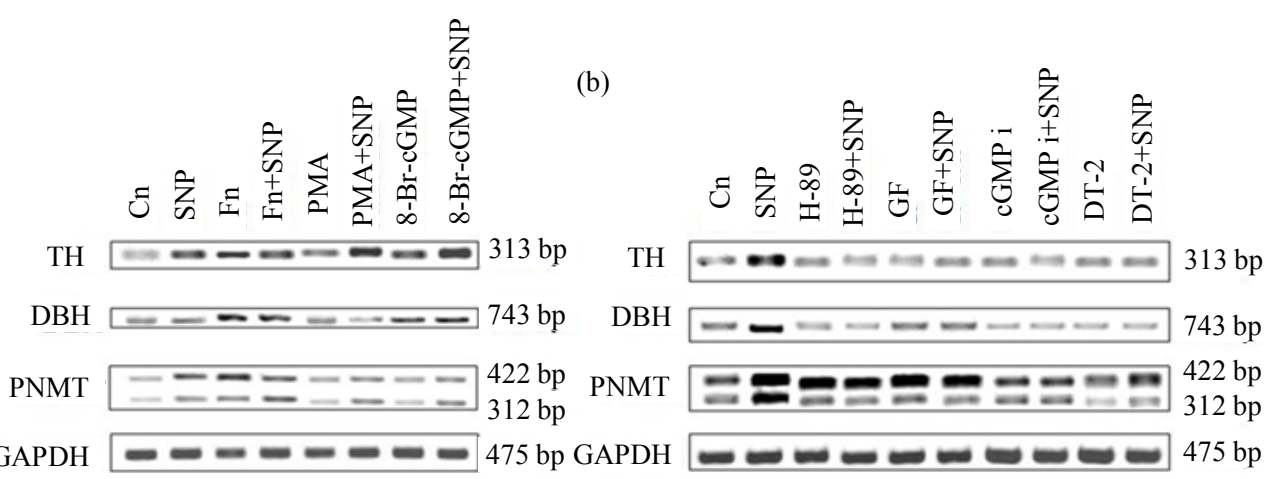

Figure 3. mRNA levels of genes associated with catecholamine biosynthesis following intracellular kinase activator, inhibitor and SNP treatments. (a) PC12 cells were treated with $10 \mathrm{mM}$ forskolin (PKA activator), $80 \mathrm{nM}$ PMA (PKA activator), $100 \mu \mathrm{M}$ 8-Br-cGMP (cGMP analogue), $100 \mu \mathrm{M}$ SNP and the combination of each drug with SNP for $6 \mathrm{~h}$ and mRNA was harvested. (b) PC12 cells were pretreated with the PKA inhibitor (30 $\mu \mathrm{M} \mathrm{H}-89)$, PKC inhibitor (100 nM GF109203X), cGMP inhibitor (200 nM A6563), PKG inhibitor (100 nM DT-2) for 30 min prior to treatment with $100 \mu \mathrm{M}$ SNP for $6 \mathrm{~h}$. Representative image $(n=3)$ of TH, DBH, PNMT (intron-retaining and intronless splice variants) mRNA levels from semi-quantitative RT-PCR. Products were resolved on 1.5\% EtBr-agarose gels. GAPDH mRNA was used as a housekeeping control.

8-Br-cGMP (cGMP-PKG activator) with $100 \mu \mathrm{M}$ SNP for $6 \mathrm{~h}$ and RT-PCR analysis performed. Fn treatment significantly increased all four genes compared to untreated control $(p<0.01$ for TH, DBH and PNMT splice variants). The addition of SNP in combination with Fn increased TH mRNA levels compared to SNP alone (1.3fold; $p<0.001)$. PMA treatment significantly increased TH and PNMT intron-retaining mRNA levels compared to untreated control $(\mathrm{p}<0.01)$. When SNP was added in combination with PMA, TH mRNA levels were further increased compared to SNP alone (1.4-fold; $\mathrm{p}<0.001$ ), whereas DBH and PNMT intron-retaining and intronless mRNA levels were unchanged. 8-Br-cGMP treatment significantly increased mRNA levels for all three genes compared to untreated control $(\mathrm{p}<0.05)$. Treatments with 8-Br-cGMP and SNP significantly increased TH, DBH and PNMT intronless mRNA levels compared to SNP alone $(1.2,1.5,1.3$-fold; $\mathrm{p}<0.05)$.

Subsequently, specific kinase inhibitors for PKA (30 $\mu \mathrm{M}$ H-89), PKC (100 nM GF109203X), cGMP (200 nM) and PKG (100 nM DT-2) were used prior to treatment of PC12 cells with SNP for $6 \mathrm{~h}$. H-89 treatment alone only decreased PNMT intron-retaining basal mRNA levels $(p<0.01)$. H-89 significantly attenuated the increase in TH, DBH and PNMT intron-retaining and intronless mRNA levels induced by SNP $(0.3,0.5,0.8,0.5$-fold; $\mathrm{p}$ $<0.05)$ compared to SNP alone. GF treatment alone altered PNMT intron-retaining mRNA levels $(\mathrm{p}<0.001)$. 
GF significantly attenuated the increase in TH, DBH and PNMT mRNA levels induced by SNP $(0.3,0.8,0.8$, 0.6-fold; $\mathrm{p}<0.05$ ) compared to SNP alone. cGMP inhibitor alone altered PNMT intron-retaining mRNA levels $(\mathrm{p}<0.05)$. cGMP inhibitor significantly attenuated the increase in TH, DBH and PNMT intronless mRNA levels $(0.4,0.6,0.5$-fold; $\mathrm{p}<0.01)$ compared to SNP alone. Lastly, DT-2 did not alter basal mRNA levels for any of the four genes. DT-2 significantly attenuated the increase in TH, DBH and PNMT intron-retaining and intronless mRNA levels $(0.4,0.6,0.5,0.5$-fold; $\mathrm{p}<0.01)$ compared to SNP alone.

\subsection{Effect of RNA Protein Synthesis Inhibitors on NO Regulation of CA Biosynthetic Enzymes}

To assess whether the mRNA induction caused by NO was associated with de novo synthesis of mRNA and/or specific protein, $50 \mathrm{nM}$ Actino-D, an inhibitor of RNA polymerase, and $1 \mu \mathrm{M}$ cycloheximide, an inhibitor of protein biosynthesis was added to media prior to treatment of cells with SNP (Figure 4). Actino-D treatment did not alter basal mRNA levels for any of the three genes. When SNP was added in combination with Actino-D, TH, DBH and PNMT intronless mRNA levels significantly decreased compared to treatments with SNP alone $(0.6,0.4,0.6$-fold; $p<0.001)$, whereas PNMT intron-retaining levels were unchanged. Cyclohex treatments did not alter basal mRNA levels for any of the three genes. The addition of SNP in combination with Cyclohex significantly decreased TH, DBH, PNMT intron-retaining and intronless $(0.5,0.6,0.7,0.6$-fold; $p<0.05)$ compared to treatments with SNP alone $(\mathrm{p}<0.001)$.

\section{Discussion}

Nitric oxide has been associated with a wide range of physiological processes and also functions as a central and peripheral neuronal messenger or neurotransmitter [3]. Studies show that NO can regulate expression of genes encoding the CA biosynthetic enzymes in bovine chromaffin cells [1]. There are several potential sources of NO influencing the adrenal medulla such as 1) endothelial cells of closely packed capillary vessels that produce NO, 2) preganglionic sympathetic fibers and 3) chromaffin cells themselves [2]. However, the exact mechanism by which NO increases gene expression of CA biosynthetic enzymes is poorly understood. This study investigated the effects of $\mathrm{NO}$ on neural and hormonal regulation of CA biosynthetic enzymes, and examined the signaling pathways involved.

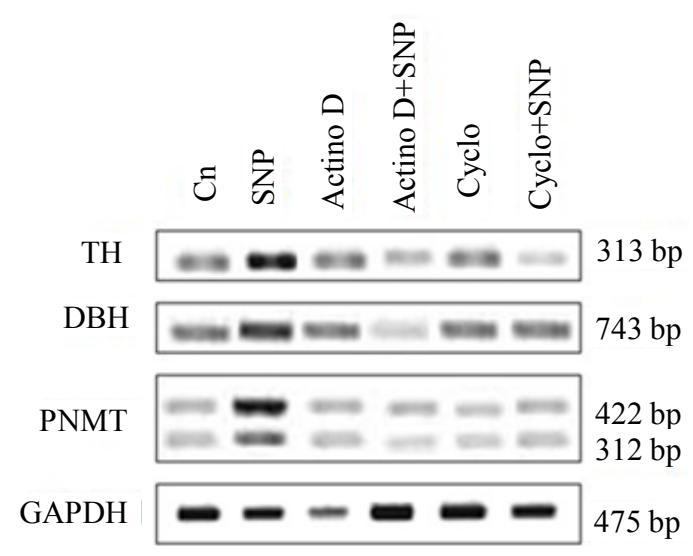

Figure 4. mRNA levels of genes associated with catecholamine biosynthesis following inhibitors of mRNA transcription and translation and SNP. PC12 cells were treated with $1 \mu \mathrm{M}$ cycloheximide, $50 \mathrm{mM}$ actinomycin D, $100 \mu \mathrm{M}$ SNP and the combination of each inhibitor with SNP for $6 \mathrm{~h}$. Representative image $(\mathrm{n}=3)$ of TH, DBH and PNMT (intron-retaining and intronless splice variants) mRNA levels from semiquantitative RT-PCR. Products were resolved on $1.5 \%$ EtBr-agarose gels. GAPDH mRNA was used as a housekeeping control. 
The in vitro PC12 cell line was used in the current study to examine the effect of SNP-derived NO on the regulation of the CA biosynthetic enzymes: TH, DBH and PNMT (intron-retaining and intronless splice variants). Both splice variants of PNMT were investigated to assess the functional implications of alternative splicing by intron-retention. Proteins translated from intron-retained mRNA species may exert a dominant negative effect on normal protein activity [11]. Significant increases in TH, DBH, PNMT intron-retaining and PNMT intronless mRNA levels were observed under SNP treatment (Figure 1), supporting previous reports in bovine adrenal chromaffin cells [1].

CA enzyme genes can be neurally regulated by the release of neurotransmitters from the splanchnic nerve and cholinergic agonists have been used to induce CA gene expression via nicotinic and muscarinic receptors [9]. To assess the effects of $\mathrm{NO}$ on cholinergic regulation of CA biosynthetic enzymes cells were treated with a combination of cholinergic agonists and SNP. In our system, further induction of TH, DBH and PNMT genes was observed following combination treatments of cholinergic agonists and NO (Figure 2(a)). This is supported by a previous report indicating that SNP-derived NO, in the presence of nicotine, increases TH, norepinephrine and epinephrine activity in the adrenal medulla [7]. Furthermore, SNP has also been shown to potentiate nicotine-induced CA secretion [12]. Our results suggest that NO can potentiate cholinergic activation of CA genes.

In addition to neural regulation, CA enzyme genes, specifically PNMT, can be regulated via hormonal stimulus such as glucocorticoids [13]. This study assessed the effects of NO on hormonal CA gene regulation via glucocorticoids. Results show further induction of TH, DBH and PNMT intronless splice variant when cells were treated with combination of glucocorticoids and SNP (Figure 2(b)); these results show additive effects of the two stimulants and thus, activation of the CA enzyme genes by NO through a glucocorticoid-dependent pathway. In support of this, glucocorticoids have been shown to increase transcription of guanosine triphosphate cyclohydrolase I (GTPCH), a rate limiting enzyme in the synthesis of NO [14]. Therefore, an increase in GTPCH by glucocorticoids could allow for further production of NO which, in turn, may lead to an increase in genes associated with CA biosynthesis.

Stimulation of the splanchnic nerve activates a number of secondary messenger systems within the adrenal medullary cells, including that of cAMP-PKA, PKC and PKG that can modulate CA gene regulation [1] [8]. Results from this study show that NO can potentiate the induction of both TH and PNMT mRNA by PKA and PKC and these responses can be completely blocked by H-89 (PKA inhibitor) and GF109203X (PKC inhibitor), respectively (Figure 3(a) and Figure 3(b)). Activation of the cAMP-PKA and PKC pathways have been shown to increase TH and PNMT transcript levels [5]. Furthermore, it has been previously shown that TH activity can be increased through parallel activation of the cGMP and cAMP-dependent protein kinase [15]. This would support a parallel activation of CA biosynthetic enzymes by the NO-mediated cGMP and cAMP-PKA pathway leading to increases in TH and PNMT transcript levels. Similar observations have been made for the PKC pathway where $\mathrm{TH}$ gene activation by neurotensin agonist requires PKC activation and NO synthesis, two distinct but complementary signaling pathways [5]. Taken together, these results suggest that further induction of the TH and PNMT genes could require both PKA and/or PKC stimulation with NO production. Moreover, treatment with the cGMP analogue in combination with SNP also significantly increased gene expression of all three enzyme genes. NO increases cGMP levels and subsequently, activation of the cGMP/PKG pathway has been shown to upregulate TH activity through phosphorylation [16]. Previous studies have suggested that effects mediated by NO on CA enzyme genes occur via the cGMP/PKG pathway [1]. Moreover, inhibition of cGMP and PKG blocked induction by NO in our system as well as in bovine chromaffin cells [1]. In addition, the RNA and protein synthesis inhibitors, actino-D and cyclohex, respectively, abrogated NO-mediated increases in TH, DBH and PNMT mRNAs, indicating that both transcription and synthesis of key transcriptional proteins are required.

In summary, the findings reported here provide evidence that NO plays an important role in CA biosynthetic enzyme gene regulation and confirms the involvement of the cGMP-dependent signaling pathway. Our studies further demonstrate that NO is capable of modulating both the glucocorticoid and cholinergic activation of adrenal CA biosynthetic enzymes, via interaction of the glucocorticoid, PKA and PKC signaling with the cGMP/ PKG signaling system.

\section{Acknowledgements}

This study was supported by funding from the Natural Sciences and Engineering Council of Canada and the Ontario Graduate Scholarship. 


\section{References}

[1] Lai, F.J., Huang, S.S., Hsieh, M.C., Hsin, S.C., Wu, C.H., Hsin, Y.C., et al. (2005) Upregulation of Neuronal Nitric Oxide Synthase mRNA and Protein in Adrenal Medulla of Water-Deprived Rats. The Journal of Histochemistry and Cytochemistry, 53, 45-53. http://dx.doi.org/10.1369/jhc.4A6350.2005

[2] Oset-Gasque, M.J., Parramon, M., Hortelano, S., Bosca, L., and Gonzalez, M. P. (1994) Nitric Oxide Implication in the Control of Neurosecretion by Chromaffin Cells. Journal of Neurochemistry, 63, 1693-1700. http://dx.doi.org/10.1046/j.1471-4159.1994.63051693.x

[3] Oset-Gasque, M.J., Vicente, S., Gonzalez, M.P., Rosario, L.M. and Castro, E. (1998) Segregation of Nitric Oxide Synthase Expression and Calcium Response to Nitric Oxide in Adrenergic and Noradrenergic Bovine Chromaffin Cells. Neuroscience, 83, 271-280. http://dx.doi.org/10.1016/S0306-4522(97)00377-1

[4] Chen, M.J. and Russo-Neustadt, A.A. (2007) Nitric Oxide Signaling Participates in Norepinephrine-Induced Activity of Neuronal Intracellular Survival Pathways. Life Sciences, 81, 1280-1290. http://dx.doi.org/10.1016/j.lfs.2007.09.003

[5] Najimi, M., Robert, J.J., Mallet, J., Rostene, W. and Forgez, P. (2002) Neurotensin Induces Tyrosine Hydroxylase Gene Activation through Nitric Oxide and Protein Kinase C Signaling Pathways. Molecular Pharmacology, 62, 647653. http://dx.doi.org/10.1124/mol.62.3.647

[6] Unsworth, B.R., Hayman, G.T., Carroll, A. and Lelkes, P.I. (1999) Tissue-Specific Alternative mRNA Splicing of Phenylethanolamine N-Methyltransferase (PNMT) during Development by Intron Retention. International Journal of Developmental Neuroscience, 17, 45-55. http://dx.doi.org/10.1016/S0736-5748(98)00058-6

[7] Kumai, T., Tanaka, M., Tateishi, T., Asoh, M. and Kobayashi, S. (1998) Effects of Sodium Nitroprusside on the Catecholamine Synthetic Pathway in the Adrenal Medulla of Rats. Japanese Journal of Pharmacology, 77, 20-210. http://dx.doi.org/10.1254/ijp.77.205

[8] Tai, T.C. and Wong, D.L. (2003) Protein Kinase A and Protein Kinase C Signaling Pathway Interaction in Phenylethanolamine N-Methyltransferase Gene Regulation. Journal of Neurochemistry, 85, 816-829. http://dx.doi.org/10.1046/j.1471-4159.2003.01728.x

[9] Wong, D.L., Anderson, L.J. and Tai, T.C. (2002) Cholinergic and Peptidergic Regulation of Phenylethanolamine NMethyltransferase Gene Expression. Annals of the New York Academy of Sciences, 971, 19-26. http://dx.doi.org/10.1111/j.1749-6632.2002.tb04428.x

[10] Serova, L.I., Nankova, B., Kvetnansky, R. and Sabban, E. L. (1997) Immobilization Stress Elevates GTP Cyclohydrolase I mRNA Levels in Rat Adrenals Predominantly by Hormonally Mediated Mechanisms. Stress (Amsterdam, Netherlands), 1, 135-144. http://dx.doi.org/10.3109/10253899709001103

[11] Wong, D.L. (2006) Epinephrine Biosynthesis: Hormonal and Neural Control during Stress. Cellular and Molecular Neurobiology, 26, 891-900. http://dx.doi.org/10.1007/s10571-006-9056-6

[12] O’Sullivan, A.J. and Burgoyne, R.D. (1990) Cyclic GMP Regulates Nicotine-Induced Secretion from Cultured Bovine Adrenal Chromaffin Cells: Effects of 8-Bromo-Cyclic GMP, Atrial Natriuretic Peptide, and Nitroprusside (Nitric Oxide). Journal of Neurochemistry, 54, 1805-1808. http://dx.doi.org/10.1111/j.1471-4159.1990.tb01238.x

[13] Wong, D.L., Tai, T.C., Wong-Faull, D.C., Claycomb, R. and Kvetnansky, R. (2004) Genetic Mechanisms for Adrenergic Control during Stress. Annals of the New York Academy of Sciences, 1018, 387-397. http://dx.doi.org/10.1196/annals.1296.048

[14] Serova, L., Nankova, B., Rivkin, M., Kvetnansky, R. and Sabban, E.L. (1997) Glucocorticoids Elevate GTP Cyclohydrolase I mRNA Levels in Vivo and in PC12 Cells. Brain Research. Molecular Brain Research, 48, 251-258.

[15] Roskoski Jr., R. and Roskoski, L.M. (1987) Activation of Tyrosine Hydroxylase in PC12 Cells by the Cyclic GMP and Cyclic AMP Second Messenger Systems. Journal of Neurochemistry, 48, 236-242.

[16] Rodriguez-Pascual, F., Miras-Portugal, M.T. and Torres, M. (1996) Effect of Cyclic GMP-Increasing Agents Nitric Oxide and C-type Natriuretic Peptide on Bovine Chromaffin Cell Function: Inhibitory Role Mediated by Cyclic GMPDependent Protein Kinase. Molecular Pharmacology, 49, 1058-1070. 